\title{
Dinâmicas colaborativas de discussão visual: um mapeamento sistemático
}

\section{Collaborative dynamics of visual discussion: a systematic mapping}

Luciana da Silva Bertoso, Reinaldo Pereira de Moraes, Stephania Padovani

representações gráficas, pensamento visual, facilitação gráfica, desenho colaborativo, desenho conjunto

Este artigo aborda, sob a ótica do Design da Informação, a prática do pensamento visual (visual thinking) por meio da elaboração de representações gráficas na organização e expressão de informações. Nesse contexto, o objetivo deste trabalho é apresentar um mapeamento sistemático sobre como as dinâmicas colaborativas de discussão visual têm sido praticadas nos últimos dez anos. Para isso, a Revisão Bibliográfica Sistemática (RBS) foi adotada como procedimento metodológico. Os termos graphic facilitation, joint drawing e collaborative drawing foram pesquisados no Portal de Periódicos da CAPES, sendo aceitos, como resultados desta busca, os artigos revisados por pares publicados a partir de 2010. Os resultados foram organizados e descritos quanto ao tipo de comunicação estabelecida entre os participantes, quantidade e faixa etária dos envolvidos na dinâmica, tipo de suporte oferecido para a elaboração da representação, áreas de conhecimento nas quais as dinâmicas foram aplicadas e como a representação gráfica foi utilizada.

graphic representation, visual thinking, graphic facilitation, collaborative drawing, joint drawing

This paper addresses, from the perspective of Information Design, the practice of visual thinking through the elaboration of graphic representations in the organization and expression of information. In this context, this work aims to present a systematic mapping on how the collaborative dynamics of visual discussion have been practiced in the last ten years. For this, a Systematic Literature Review (SLR) was adopted as a methodological procedure. The terms graphic facilitation, joint design and collaborative design were searched on the Portal of Journals of the CAPES, and were accepted, as the results of this search, the peer-reviewed papers published from 2010. The results were organized and described in terms of the type of communication between the participants, the quantity and age range of people involved in dynamics, the type of support offered for the elaboration of the representation, areas of knowledge in which the dynamics were applied and how graphic representation was used.

\section{Introdução}

Na maioria das atividades escolares/acadêmicas os estudantes precisam compreender, analisar e sintetizar conteúdos. Nesse sentido, o resumo (síntese) é uma estratégia cognitiva de aprendizagem na qual o aluno organiza, armazena e elabora informações (Moreira et al., 2016). Elaborar explicações visuais exige a reorganização dos modelos mentais do estudante por meio do refinamento das conexões entre os elementos e conceitos, promovendo um processamento profundo do material e um modelo mental mais abrangente (Bobek \& Tversky,

Anais do $10^{\circ} \mathrm{CIDI}$ e $10^{\circ} \mathrm{CONGIC}$

Kelli C.A.S. Smythe, Rafael de Castro Andrade (orgs.)

Sociedade Brasileira de Design da Informação - SBDI

Curitiba | Brasil | 2021
Proceedings of the $10^{\text {th }} \mathrm{CIDI}$ and $10^{\text {th }} \mathrm{CONGIC}$

Kelli C.A.S. Smythe, Rafael de Castro Andrade (orgs.)

Sociedade Brasileira de Design da Informação - SBDI Curitiba | Brazil | 2021 
2016; Craik \& Lockhart, 1972). Essas explicações visuais resultam do pensamento visual (visual thinking), no qual o conteúdo de diálogos, discursos, reuniões e de outras situações, são organizados e registrados por meio de representações gráficas (Arnheim, 1969; Mckim, 1980; Cyrs, 1997; Roam, 2009).

A criação colaborativa de representações gráficas entre um grupo de estudantes demanda uma interação que pode ser promovida pelas Representações Gráficas de Síntese (RGS) (Padovani \& Heemann, 2016). As RGSs surgiram como uma estratégia pedagógica para aproveitar as habilidades de análise, produção e avaliação de representações gráficas dos estudantes, tornando-se, posteriormente, uma dinâmica que possibilita uma reflexão profunda sobre os conteúdos representados (Padovani, 2012).

Em revisões de literatura sobre RGSs e dinâmicas similares, foram encontrados estudos que utilizam nomenclaturas associadas aos temas graphic facilitation, joint drawing e collaborative drawing. Todos se baseiam em dinâmicas colaborativas de discussão visual envolvendo representações gráficas na comunicação ou expressão de informações. Dinâmicas colaborativas de discussão visual são entendidas neste artigo como aqueles momentos nos quais dois ou mais indivíduos estão presentes em um ambiente físico ou virtual interagindo ativamente de forma síncrona ou assíncrona no exercício do pensamento visual.

Diante do exposto, este artigo tem o objetivo de apresentar um mapeamento sistemático sobre como as dinâmicas colaborativas de discussão visual têm sido praticadas nos últimos dez anos (conforme relatado na literatura científica internacional). Para tanto, foi desenvolvida uma Revisão Bibliográfica Sistemática (RBS) no Portal de Periódicos da Coordenação de Aperfeiçoamento de Pessoal de Nível Superior (CAPES). Visamos verificar o tipo de comunicação estabelecida entre os participantes, quantidade e faixa etária dos envolvidos na dinâmica, tipo de suporte oferecido para a realização da representação, áreas de conhecimento nas quais as dinâmicas foram aplicadas e como a representação gráfica foi utilizada.

\section{Contexto teórico}

\section{Pensamento visual}

O pensamento visual (visual thinking) se refere à visualização de informações através das imagens (Seels, 1994) e permeia todas as atividades humanas, desde as abstratas e teóricas até as práticas e cotidianas. Não é um modo de pensar exclusivo dos artistas ou designers, visto que cirurgiões também pensam visualmente para realizar uma operação, químicos para construir modais moleculares, matemáticos para considerar relações abstratas de espaçotempo, engenheiros para projetar circuitos, empresários para organizar e programar seus trabalhos (McKim, 1980). Observa-se nessas práticas que o pensamento visual pode nos auxiliar na resolução de problemas, descobrindo, compartilhando e desenvolvendo ideias de forma rápida e intuitiva por meio de imagens (Roam, 2009).

Nesse contexto, três estratégias interativas de pensamento são observadas: visualizar, imaginar e desenhar (Mckim, 1980; Cyrs, 1997) (Figura 1). Visualizar envolve a percepção 
visual de objetos bi ou tridimensionais e suas relações com as experiências anteriores do observador. Imaginar envolve criar imagens mentais que podem se referir à realidade ou realidades alternativas. Desenhar envolve expressar a ideia através de alguma forma visual (imagens, esculturas, pinturas, rabiscos). Na prática, o indivíduo que está operando o pensamento visualmente utiliza a visualização, imaginação e o desenho de uma forma fluida e dinâmica, passando de um tipo de imagem para outro, fazendo um ciclo entre as imagens percebidas (visualizar), internas (imaginar) e gráficas (desenhar), até que o problema seja resolvido (McKim, 1980).

Figura 1: Estratégias de pensamento, adaptado de Cyrs (1997) e McKim (1980)



Essa experiência visual é um processo de construção de conhecimento no qual abstrações, significados, conteúdos, conceitos e atividades podem ser expressados visualmente por meio de representações gráficas. Entende-se representação gráfica como um artefato visível registrado em superfície mais ou menos plana e que tenha sido elaborado a fim de expressar algum tipo de informação (Engelhardt, 2002).

Para elaborar essas representações, não é necessário ter a habilidade de desenhar altamente treinada, pois qualquer pessoa é capacitada para se expressar visualmente através de representações gráficas (Gray, 2012). Além disso, na medida em que o pensamento visual se disseminou, ferramentas que fornecem imagens prontas para apoiar o diálogo em grupos, tais como modelos gráficos, softwares para criação de mapas mentais e picture cards, foram desenvolvidas (Sibbet, 2013) e podem auxiliar as pessoas na tarefa de representar (Cyrs, 1997).

Na prática, o pensamento visual tem sido materializado por meio da realização de dinâmicas colaborativas de discussão visual. Em nossa revisão de literatura, verificamos que muitos estudos se baseiam em dinâmicas colaborativas de discussão visual nomeadas e descritas como facilitação gráfica, desenho colaborativo e desenho conjunto.

\section{Facilitação gráfica (graphic facilitation)}

A representação visual dos pensamentos e discussões elaborados por um grupo de pessoas é chamada de facilitação gráfica (Sibbet, 2001). Essas contribuições são registradas na frente do 
grupo e essa prática incentiva uma maior participação nas reuniões. O facilitador gráfico ouve as conversas, traduz estes dados verbais e não verbais em forma de imagens visuais a fim de sintetizar e integrar o pensamento individual e grupal. A facilitação gráfica também acomoda diversos estilos de aprendizagem e processamento da informação possibilitando melhor entendimento dos assuntos discutidos (Gardner, 1985).

Nesse sentido, Ravetz e Ravetz (2017) exemplificam que a facilitação gráfica é uma técnica importante para workshops focados em processos e o método de sinergia visual trabalha o material visual (de dentro ou fora do local) como um catalisador do pensamento de grupo. Com base nesses exemplos e em outras observações, eles propõem uma classificação de três vertentes paralelas para as representações gráficas do pensamento visual:

(a) visualização no processo - usada em workshops ou situações de discussão nos quais se trabalha a construção de consenso, a mediação de conflitos e o estabelecimento de estratégias;

(b) visualização do processo - na captura direta do diálogo, do debate, da discussão e/ou do conflito;

(c) visualização como processo - na qual são levantados tópicos de interesse público, dando voz e visibilidade às comunidades.

Compreender como as representações gráficas têm sido utilizadas em dinâmicas colaborativas de discussão visual tem sua importância porque as técnicas de facilitação gráfica têm sido implantadas em diferentes contextos organizacionais e educacionais. The World Café e Circle of Courage são exemplos de variações da aplicação da facilitação gráfica.

\section{The World Café}

A técnica The World Café traz uma abordagem de facilitação gráfica considerada mais rápida e profunda (Brown \& Isaacs, 2005) convidando à participação pessoas que não são especialistas em representação gráfica. Para a realização da dinâmica, os pensamentos são compartilhados em grupos de 4 a 5 pessoas na etapa 1 e cada grupo compartilha seus conhecimentos com os demais grupos enquanto o facilitador gráfico elabora a representação visual sintetizando o pensamento geral na etapa 2 (Prewitt, 2011).

\section{Circle of Courage}

O Circle of courage foi sistematizado por Brendtro et al. (2002) como uma resposta aos jovens com distúrbios emocionais e de comportamento a partir dos conhecimentos que as tribos indígenas da américa dominavam para enfrentar a vida. Do ponto de vista representacional, o Circle of Courage é uma apresentação visual formada por quadrantes representativos dos quatro princípios de desenvolvimento positivo da juventude (pertencimento, domínio, independência e generosidade) a fim de auxiliar as pessoas a visualizarem seus sonhos e expectativas de vida (Espiner \& Hartnett, 2016). Além da pessoa envolvida nesse processo de 
desenvolvimento pessoal, outras pessoas são convidadas a se envolverem, tais como seus familiares, o professor e o conselheiro educacional da escola.

\section{Desenho conjunto (joint drawing)}

A técnica de desenho conjunto é mais comumente usada na arteterapia para avaliação e intervenção com crianças e adultos e se caracteriza pela participação de duas pessoas desenhando juntas na mesma folha de papel. Ao longo dos anos tem sido aplicada com clientes e terapeutas em terapias individuais, com um casal em terapia de casais, com a criança e um de seus pais em terapia familiar e entre dois participantes em terapias de grupo (Snir \& Wiseman, 2010). Normalmente é solicitado aos participantes que realizem um desenho conjunto sem comunicação verbal entre eles. O terapeuta faz o papel de facilitador da dinâmica e raramente participa da execução do desenho (Rosen et al., 2016). O princípio dessa prática se baseia no fato de que desenhar em uma folha compartilhada pode revelar temas interpessoais e características significativas dos participantes (Snir \& Wiseman, 2010). Embora a organização e condução mais comum de uma dinâmica de desenho conjunto seja por meio do desenho livre existem outras derivações a partir desta técnica, tais como Joint Painting Procedure, Interactive Drawing Task e Developmental Transformation Art Therapy.

Joint Painting Procedure (JPP)

A dinâmica JPP foi desenvolvida por Gavron (2013) a partir de mais de 15 anos de experiência com arteterapia aplicada em duplas de pais e filhos na meia infância (6 a 12 anos). É um processo no qual os dois parceiros pintam juntos no mesmo pedaço de papel seguindo cinco etapas: (1) os dois marcam a lápis um espaço pessoal no papel; (2) ambos pintam em seus próprios espaços, um ao lado do outro, (3) criam uma moldura em volta do espaço pessoal, (4) pintam um caminho do seu espaço pessoal até o espaço do outro participante e (5) pintam juntos na área compartilhada do papel (Gavron, 2013).

Interactive Drawing Task (IDT)

A IDT foi desenvolvida por van Ommeren e Begeer e conforme Lai et al. (2020), a realização dessa dinâmica permite a interação espontânea com o facilitador da dinâmica a fim de atingir um objetivo em comum (desenhar juntos). De acordo com esses autores, para a realização desta dinâmica são necessários os seguintes materiais: uma mesa, cartolina tamanho A3, quatro marcadores coloridos (vermelho, azul, preto, verde) e uma câmera de vídeo. A dinâmica se inicia a partir da única instrução: "Vamos desenhar juntos, escolha um marcador". O facilitador deve ser recíproco com o desenho do outro participante, interagindo e completando o desenho, mas também estimulando que o outro participante contribua na execução do desenho iniciado pelo facilitador. 
Developmental Transformation Art Therapy (DvT)

A arteterapia DvT adota o desenho conjunto em papel com a participação direta do terapeuta. Essa abordagem utiliza 4 técnicas como resposta do terapeuta ao desenho do cliente: espelhamento, renderização fiel, renderização emergente e renderização divergente (Rosen, 2016). No espelhamento, o terapeuta responde ao comportamento do cliente replicando o desenho realizado. Na renderização fiel, o terapeuta desenha na cena algo que seja compatível com o que foi desenhado pelo cliente. Na renderização emergente, o terapeuta finaliza a representação de um elemento expressivo que está emergindo, mas não totalmente formado. Na renderização divergente, o terapeuta desenha elementos antagônicos aos elementos apresentados pelo cliente.

\section{Desenho colaborativo (collaborative drawing)}

O desenho colaborativo vem sendo utilizado como dinâmica colaborativa de discussão visual em diversas áreas, tais como Educação, Psicologia, Negócios e Design e consiste em duas ou mais pessoas criarem uma representação gráfica. Porém em cada área há uma abordagem teórica diferente com técnicas próprias. Alguns exemplos destas técnicas são: Collaborative Drawing Technique (CDT), Six-Member Collaborative Drawing Technique (S-CDT), Intergenerational collaborative drawing (ICD), Rich Picture (RP), Collaborative concept maps (mapas conceituais colaborativos) e Squiggle Game.

\section{Collaborative Drawing Technique (CDT)}

Foi criada por Smith (1985) no campo da psicoterapia familiar. É uma tarefa interativa não verbal aplicada a uma família e observada por um terapeuta para compreender os padrões, dinâmicas e hierarquias presentes nos sistemas familiares (Smith, 1985). Os materiais utilizados são papel, canetas coloridas e cronômetro, uma mesa na qual o papel ficará e uma cadeira. O desenho colaborativo não é realizado simultaneamente. Após ser definida uma ordem, cada membro da família em sua vez deve se sentar na cadeira, realizar o desenho e sair para que o próximo membro continue. É realizado em rodadas decrescentes de tempo iniciando com 30 segundos e diminuindo para 20,15, 10, 5 e finalizando com 3 segundos (Smith, 1985). O resultado final da tarefa é um registro permanente e visível da interação familiar. Como cada indivíduo usa seu próprio giz de cera com cores distintas, as contribuições de cada participante são facilmente discerníveis (Smith, 1985).

\section{Six-Member Collaborative Drawing Technique (S-CDT)}

Foi introduzida por Joohyung Park (Park \& Kang, 2018). O S-CDT envolve avaliação estruturada de arteterapia, em que um grupo de seis pessoas desenha um cenário comunitário em uma única folha grande de papel. Os materiais usados no S-CDT são um papel branco tamanho B1, uma caneta preta e um pacote com giz de cera colorido. A conclusão do teste leva 45 minutos e inclui três etapas: (1) desenho individual, (2) desenho colaborativo e (3) 
definição do nome da comunidade desenhada. Ao final da dinâmica, os elementos formais dos desenhos são usados para medir a tendência colaborativa.

\section{Squiggle Game}

Jogo de desenho colaborativo, criado pelo pediatra/psiquiatra infantil Winnicott para uso com crianças no processo terapêutico com objetivo de construir relacionamento e melhorar a comunicação. $O$ jogo é realizado em duplas, no qual um dos participantes (o terapeuta e a criança, ou duas crianças) faz um rabisco na página e o outro participante transforma o rabisco em uma representação. Como resultado, o processo promove proximidade e alinhamento afetivo entre os envolvidos. Por essa abordagem o jogo vem sendo usado em múltiplos cenários para apoiar a construção de relacionamentos (Sakr, 2019).

Intergenerational collaborative drawing (ICD)

É um método de pesquisa em educação baseada em artes (Arts-Based Education Research) no qual as práticas artísticas são usadas para investigar questões e assuntos educacionais explorando a potencialidade do desenho para o ensino e pesquisa na educação infantil. O ICD envolve adultos, crianças e/ou pesquisadores desenhando ao mesmo tempo no mesmo papel (Knight et al., 2015).

\section{Rich Picture (RP)}

É uma ferramenta desenvolvida por Peter Checkland, nos anos 1980, como parte da Soft Systems Methodology (SSM) (Bell \& Morse, 2010; Brouwer et al., 2019) e tem sido utilizada para resolução colaborativa de problemas em outras áreas como sustentabilidade, empreendedorismo social, visualização da informação e representação do conhecimento, a partir do uso colaborativo da representação gráfica (Brouwer et al., 2019). A dinâmica pode ser realizada à mão ou em ferramentas digitais. Quando realizada manualmente, um único papel deve ser utilizado por todo o grupo (Bell \& Morse, 2010). Um participante assume o papel de facilitador para estimular os demais a contribuírem independente das habilidades de desenho. No centro da folha/tela deve ser desenhada a situação problemática, a partir da qual serão identificados as partes interessadas e suas relações. Textos podem ser utilizados para criar legendas, evitando o uso em textos longos (Brouwer et al., 2019).

\section{Collaborative concept maps (mapas conceituais colaborativos)}

Os mapas conceituais são ferramentas gráficas para a organização e representação do conhecimento, incluem conceitos, geralmente dentro de círculos ou quadrados, e relações entre conceitos, que são rotuladas e indicadas por linhas que os interligam (Novak \& Cañas, 2006). Os mapas conceituais colaborativos são utilizados como ferramenta educacional para alunos de várias idades e em diversas situações de aprendizagem, sendo feitos à mão e/ou com apoio de dispositivos digitais (Islim, 2018). 


\section{Procedimentos metodológicos}

Uma Revisão Bibliográfica Sistemática (RBS) foi conduzida a partir do roteiro proposto por Conforto et al. (2011). A base de dados para a pesquisa foi o Portal de Periódicos da Coordenação de Aperfeiçoamento de Pessoal de Nível Superior (CAPES). Esta pesquisa se propôs a responder como as representações gráficas têm sido utilizadas no contexto comunicacional colaborativo, quais nomenclaturas têm sido adotadas e quais os públicos-alvo na aplicação destas dinâmicas. Durante o desenvolvimento da fundamentação teórica deste trabalho, observamos que os termos graphic facilitation, collaborative drawing e joint drawing têm sido usados para descrever dinâmicas colaborativas de discussão visual. Por isso, na RBS realizada, estes três termos foram adotados como strings de busca.

Tradicionalmente, o filtro 1 (F1) é aplicado na leitura de título, palavras-chaves e resumo, o filtro 2 (F2) é aplicado na leitura da introdução e da conclusão do artigo e o filtro 3 (F3) é aplicado na leitura completa do texto a fim de cumprir com os critérios de inclusão e exclusão descritos na figura 2 (Conforto et al., 2011).

Figura 2: Filtros e critérios de inclusão e exclusão. Fonte: os autores (2021)

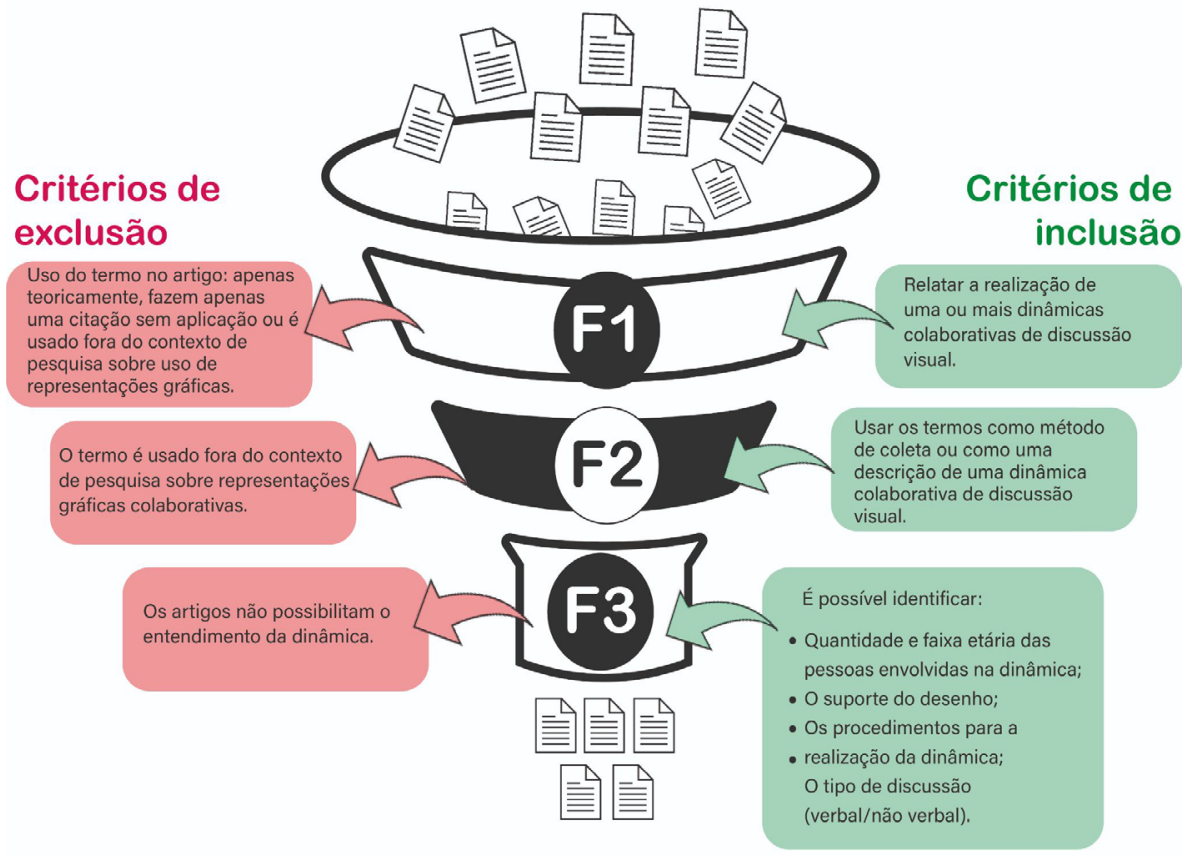

Entretanto, foi observado que alguns artigos traziam resultados interessantes para esta pesquisa sem apresentar palavras-chaves e/ou resumo em seu conteúdo. Por isso, os filtros F1 e F2 demandaram, além do título, palavras-chaves e resumo, a leitura dos procedimentos metodológicos dos artigos, e o F3 foi aplicado a partir da leitura de todo o texto.

No portal Periódicos, a busca foi realizada a partir da funcionalidade de busca simples por assunto. As três buscas foram efetuadas separadamente entre os dias 10 e 16 de abril de 2021. Para as três strings foram definidos como resultados de interesse os artigos revisados 
por pares e publicados entre 2010 e 2021. O processo de leituras e filtragens dos artigos está representado na figura 3 .

Figura 3: Esquematização do processo de filtragem de artigos da RBS. Fonte: os autores (2021)

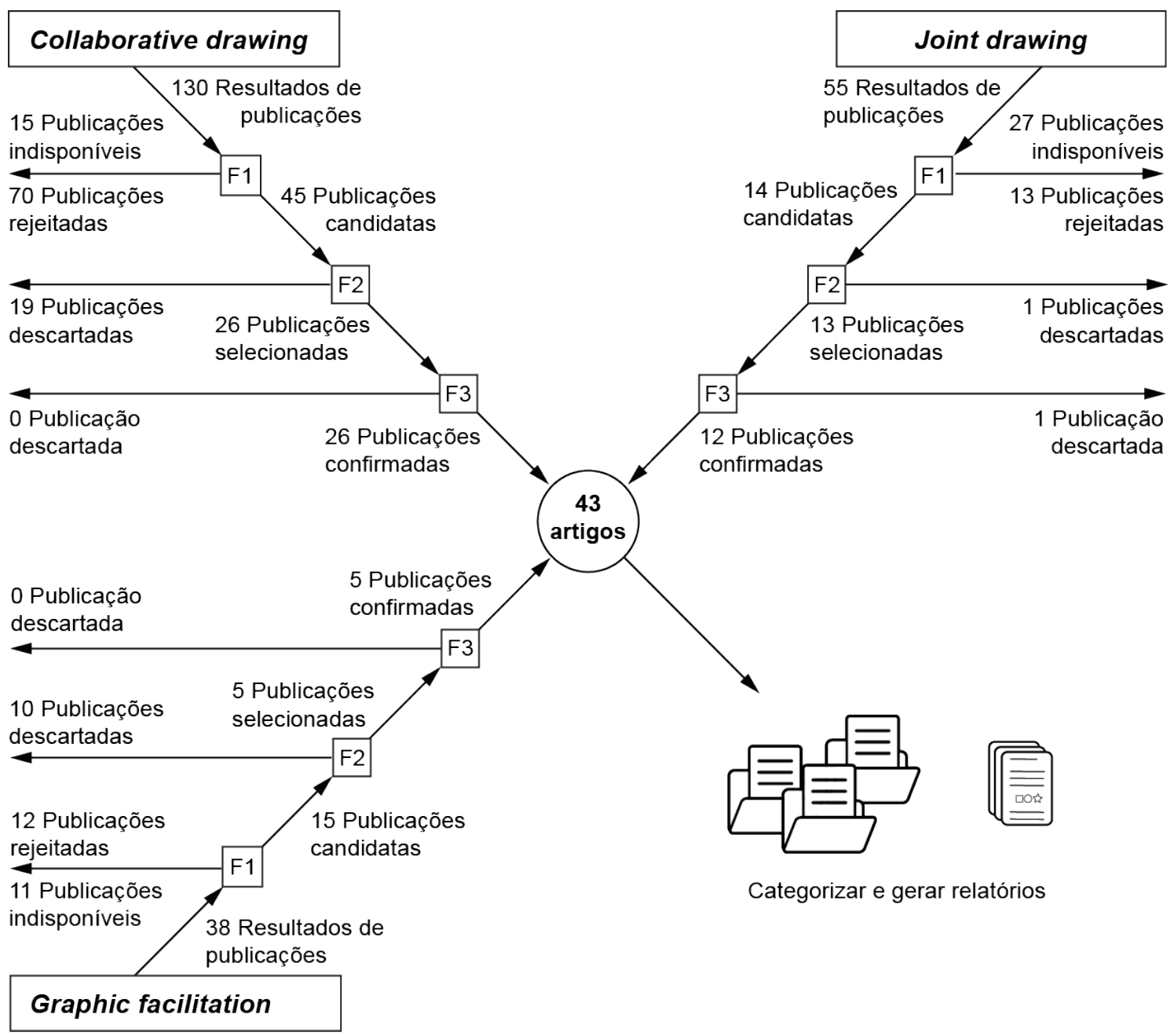

A busca pela string collaborative drawing resultou em 130 artigos, entretanto, apenas 115 permitiram acesso e download. A leitura realizada para a avaliação do filtro 1 aprovou 45 artigos. A leitura e aplicação do Filtro 2 aprovou 26 artigos, os quais foram confirmados pela leitura completa e aplicação do filtro 3.

A busca pela string graphic facilitation resultou em 38 artigos, entretanto, apenas 27 permitiram acesso e download. A leitura realizada para a avaliação do filtro 1 aprovou 15 artigos. A leitura e aplicação do Filtro 2 aprovou 5 artigos, os quais foram confirmados pela leitura completa e aplicação do filtro 3.

A busca pela string joint drawing resultou em 55 artigos, entretanto, apenas 28 permitiram acesso e download. A leitura realizada para a avaliação do filtro 1 aprovou 14 artigos. A leitura e aplicação do Filtro 2 aprovou 13 artigos. Por fim, a leitura final e aplicação do filtro 3 aprovou 12 artigos. Os 43 artigos resultantes desse processo de filtragem foram revistos e analisados a partir das categorias descritas na figura 4 . 
Figura 4: Categorias de análise. Fonte: os autores (2021)

A. $\begin{aligned} & \text { Nomenclaturas } \\ & \text { Identificar os termos utilizados ao longo dos } \\ & \text { artigos para denominação da dinâmica de } \\ & \text { discussão e colaboração visual }\end{aligned}$

D.

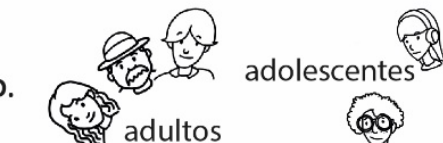

\section{कि}



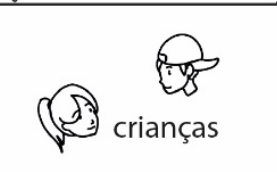

Faixa etária dos participantes

Descrever a faixa etária dos participantes em crianças, adultos, adolescentes e ambos

$\begin{array}{ll}\text { Tipo de suporte } & \begin{array}{l}\text { Identificar qual o tipo de suporte utilizado } \\ \text { para criar as representações: papel, } \\ \text { computador, tablet e ambos }\end{array}\end{array}$

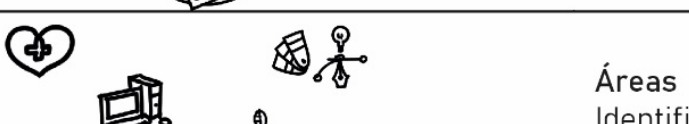
F. Identifica
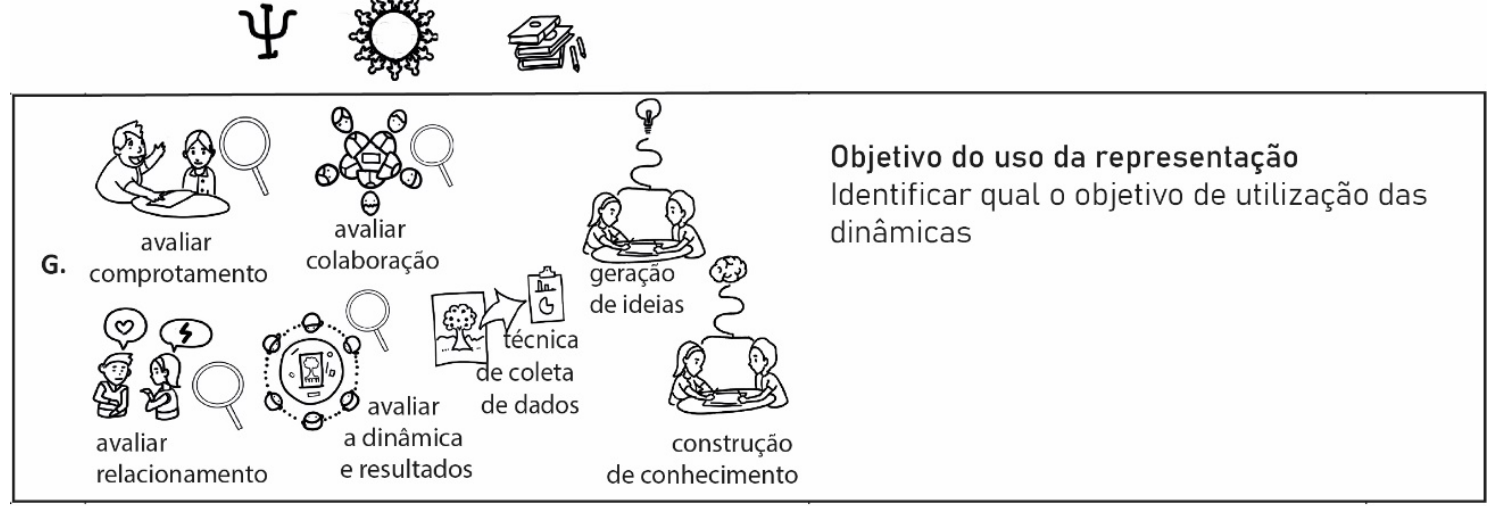

Vertente do pensamento visual

Identificar qual o propósito da elaboração da representação: visualização no processo, do processo ou como processo

\section{Resultados e discussão}

A análise dos artigos a partir das categorias mencionadas anteriormente permitiu o mapeamento sistemático e a identificação de como as dinâmicas colaborativas de discussão visual têm sido usadas e quais as suas características. Esse mapeamento pode ser observado na figura 5 . 
Figura 5: Mapeamento das dinâmicas colaborativas de discussão visual. Fonte: os autores (2021)

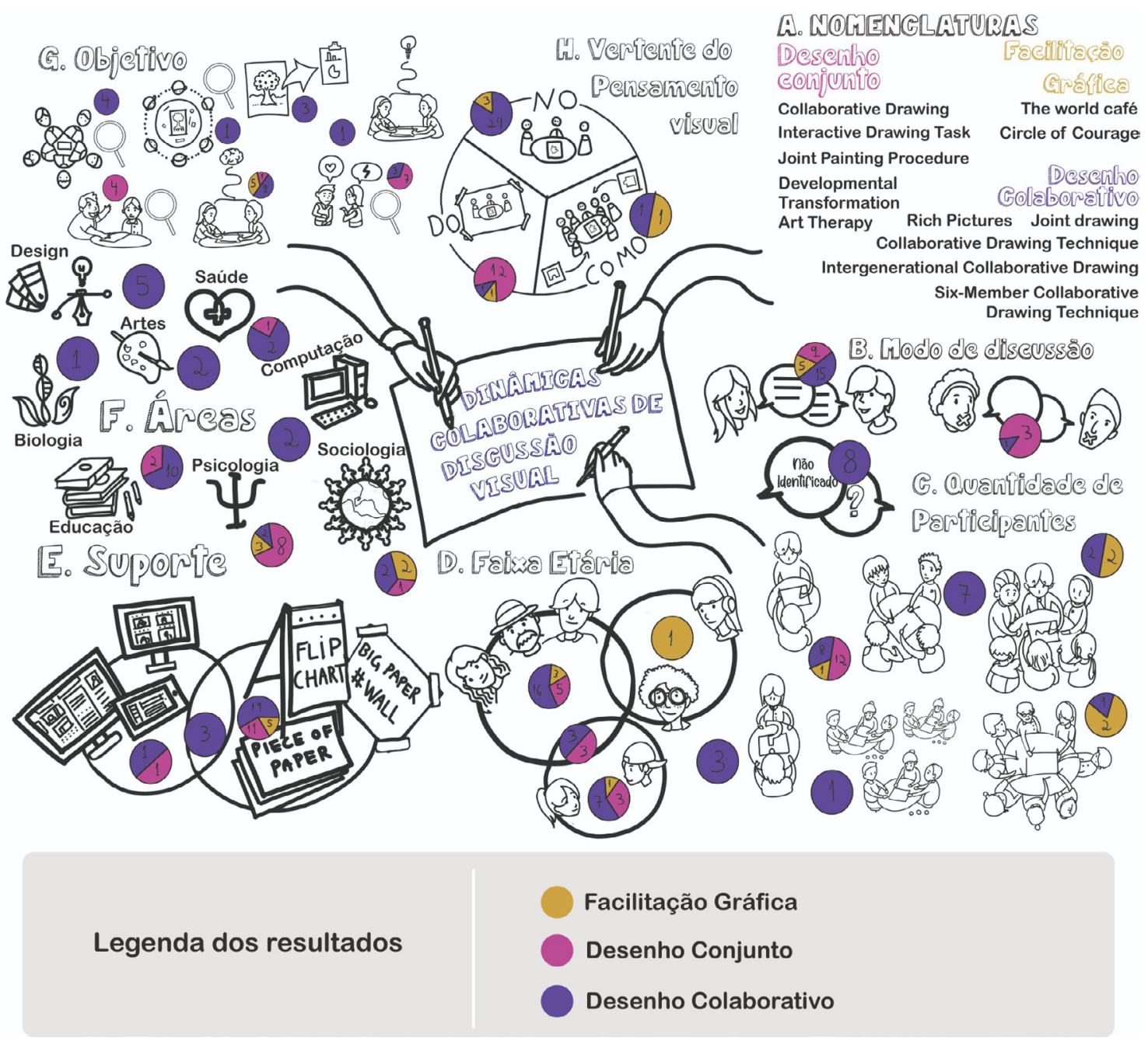

\section{Facilitação gráfica}

Embora o termo pesquisado na RBS tenha sido graphic facilitation, três dos cinco artigos confirmados pelo filtro 3 se apoiam em outras duas técnicas de discussão visual colaborativa denominadas de Circle of Courage ( 2 artigos) e The World Café (1 artigo).

Também foi observado nos cinco artigos que a discussão do assunto representado ocorre verbalmente entre os participantes e todos os desenhos foram realizados sobre papel.

A quantidade de participantes durante a dinâmica é variável e não está relacionada diretamente à técnica utilizada; dois artigos apresentaram a realização de dinâmicas envolvendo de seis a dez participantes, um artigo descreve a participação de dois participantes, enquanto outros dois descrevem a participação de mais de dez participantes.

A faixa etária dos participantes também é variável e independente da técnica aplicada. Três artigos relataram dinâmicas aplicadas com adultos, enquanto um artigo descreveu a aplicação da dinâmica com uma criança e outro com um adolescente.

Em todos os cinco textos, o objetivo da discussão visual se apoia na construção de conhecimento. Entretanto, os três artigos que descrevem a aplicação da dinâmica na área da 
Psicologia ou Terapia abordam a construção de autoconhecimento, enquanto os outros dois artigos com abordagem sociológica descrevem a construção do conhecimento organizacional.

Do ponto de vista das vertentes do pensamento visual, enquanto três artigos aplicam a facilitação gráfica como uma visualização no processo, um artigo faz sua aplicação como uma visualização do processo e outro faz a aplicação da facilitação gráfica como processo.

\section{Desenho conjunto}

A aplicação da dinâmica Joint Drawing é descrita em sete artigos. O termo joint drawing possibilitou também identificar outras técnicas de discussão visual similares ou derivadas. Dois artigos usam uma dinâmica denominada Joint Painting Procedure, enquanto um artigo aborda o uso da dinâmica Collaborative Drawing, um artigo descreve a realização da dinâmica Interactive Drawing Task, e por fim, outro artigo relata a aplicação da Developmental Transformation Art Therapy.

Foi observado que, em 9 dos 12 artigos, a discussão do assunto representado ocorreu verbalmente entre os participantes. Também pode ser observado que apenas um artigo relatou o uso de computador como suporte para a realização dos desenhos durante a realização da dinâmica e 11 dos artigos relataram a realização dos desenhos em papel.

Todos os artigos relataram a realização de dinâmicas com duplas de participantes. Em cinco artigos, as duplas eram formadas por adultos. Em três artigos, as duplas eram formadas por crianças. Outros três artigos relataram a realização de dinâmicas com duplas formadas por um adulto e uma criança. Apenas um artigo relatou a aplicação da dinâmica com duplas de adolescentes.

A maioria dos artigos (7 de 12) descrevem dinâmicas de discussão visual apoiadas na avaliação de relacionamentos. Outros quatro relatam a realização de dinâmicas a fim de avaliar comportamentos, enquanto um artigo aborda a construção de conhecimento.

Quanto às áreas de aplicação, 8 artigos estão relacionados à Psicologia ou Terapia. Dois estão inseridos na área de Educação, enquanto um está relacionado à sociologia e outro relacionado à Saúde.

Do ponto de vista das vertentes do pensamento visual, todos os 12 artigos aplicam a facilitação gráfica como uma visualização do processo.

\section{Desenho colaborativo}

A dinâmica de desenho colaborativo foi abordada em vinte e seis artigos, nos quais foi possível identificar outras técnicas de discussão visual similares ou derivadas. Três artigos utilizaram a técnica Rich Pictures em suas pesquisas e três artigos utilizam o joint drawing, enquanto dois artigos abordaram o desenho colaborativo por meio dos Collaborative Concept Maps. Apenas um artigo apresentou um estudo com a técnica Intergenerational collaborative drawing, um artigo descreveu a Six-Member Collaborative Drawing Technique e um artigo utilizou a Collaborative Drawing Technique em sua investigação. Além disso, foi identificado que no 
mesmo artigo ao se referir ao desenho colaborativo também utilizaram o termo joint drawing em três artigos.

Sobre o tipo de discussão durante a colaboração visual, foi possível identificar que em mais da metade (15 artigos) havia discussão verbal, em um artigo a discussão foi não verbal e em oito não foi possível identificar se havia ou não discussão verbal.

Em relação à quantidade de pessoas que estavam colaborando durante a dinâmica, em apenas um artigo houve a participação de mais de dez pessoas, em dois artigos colaboraram de 6 a 10 pessoas, em sete artigos colaboraram até 5 pessoas, em 8 artigos a participação foi em dupla, um artigo relatou que os participantes puderam formar grupos livremente e em três artigos não foi possível identificar a quantidade de participantes.

O foco da representação foi variado nas dinâmicas de desenho colaborativo, o maior enfoque foi na construção de conhecimento (9 artigos), quatro avaliaram a colaboração, três utilizaram o desenho colaborativo como instrumento de coleta de dados, enquanto outros três avaliaram o comportamento por meio da dinâmica. Um artigo analisou a dinâmica em si e seus resultados e mais um artigo utilizou a dinâmica como técnica para geração de ideias.

Quanto à faixa etária, mais da metade dos participantes eram adultos (16 artigos). Em sete artigos, crianças realizaram desenhos colaborativos e em outros sete, adultos e crianças trabalharam em conjunto.

Em relação às áreas, foi possível identificar que o desenho colaborativo não é específico de um campo de conhecimento. Foram identificadas dez pesquisas em Educação e cinco em Design. Artes, Psicologia, Computação, Saúde e estudos em Sociedade tiveram 2 artigos cada, enquanto uma pesquisa foi realizada na área da Biologia.

O suporte de realização do desenho colaborativo que teve maior predominância foi o papel, utilizado nas dinâmicas de 19 artigos. Três artigos descreveram o uso de papel e computador/tablet e apenas um relatou o uso de Tablet. Em apenas um artigo não foi possível identificar qual foi o suporte utilizado.

E por fim, a vertente do pensamento visual mais identificada foi a visualização no processo, na qual a representação gráfica auxiliou os participantes a discutirem conteúdos, apresentar pontos de vista, compreender comportamentos, entre outros. Já a visualização do processo foi identificada em um artigo, enquanto a visualização como processo foi observada em outro.

Tanto nos artigos envolvendo a facilitação gráfica quanto naqueles sobre desenho conjunto e desenho colaborativo, foram identificadas dinâmicas similares identificadas por nomenclaturas específicas. Sobre o modo de discussão houve a predominância do modo verbal apoiando a discussão visual. A colaboração em duplas foi mais observada no desenho conjunto e no desenho colaborativo, enquanto dinâmicas com grupos maiores foram mais observadas na facilitação gráfica. Nos três tipos de dinâmicas, mais da metade dos participantes eram adultos. Papel foi o suporte mais utilizado para criar as representações gráficas, alguns outros estudos usaram computadores para a realização das dinâmicas. Apesar das áreas identificadas serem diversas, as pesquisas em Educação totalizaram 12 artigos. 
Além disso, o objetivo mais recorrente para aplicação das dinâmicas foi a construção de conhecimento. Quanto à vertente do pensamento visual, a visualização no processo teve maior recorrência, nas quais as representações gráficas possibilitaram a visualização de consensos, mediação de conflitos e estabelecimento de estratégias.

\section{Conclusões e desdobramentos}

O presente artigo teve como objetivo apresentar um mapeamento sistemático sobre como as dinâmicas colaborativas de discussão visual têm sido praticadas nos últimos dez anos em contexto internacional. A partir da revisão de literatura sistemática no Portal de Periódicos da CAPES foi possível identificar que o pensamento visual pode ser um meio de expressar e compartilhar ideias colaborativamente, por meio de representações gráficas, criadas em dinâmicas colaborativas de discussão visual nomeadas e descritas como: facilitação gráfica, desenho colaborativo e desenho conjunto.

A RBS possibilitou identificar e categorizar 43 pesquisas que tratam dessas dinâmicas em diversos contextos resultando em um mapeamento descrito em oito categorias. O mapeamento ilustrou os resultados de cada dinâmica que foram comparados em cada categoria.

Como desdobramentos da presente pesquisa, vislumbra-se pesquisas específicas sobre dinâmicas colaborativas de discussão visual aplicadas em contexto da educação superior e no contexto da Engenharia de Requisitos para o desenvolvimento de software.

\section{Agradecimento}

À Universidade Federal do Paraná (UFPR) e ao Programa de Pós-Graduação em Design (PPGDesign). O presente trabalho foi realizado com apoio da Coordenação de Aperfeiçoamento de Pessoal de Nível Superior - Brasil (CAPES) - Código de Financiamento 001.

\section{Referências}

Arnheim, R. (1969). Visual thinking. Berkeley: University of California Press.

Bell, S., \& Morse, S. (2010). Rich pictures: a means to explore the 'Sustainable Group Mind'. In: The 16th annual international sustainable development research conference, 30 May - 01 Jun 2010, Hong Kong, China.

Bobek, E., \& Tversky, B.(2016). Creating visual explanations improves learning. Cogn. Research 1, 27. https://doi.org/10.1186/s41235-016-0031-6

Brendtro, L., Brokenleg, M., \& van Bockern, S. (2002). Reclaiming youth at risk: Our hope for the future. Bloomington, IN: Solution Tree.

Brown, J., \& Isaacs, D. (2005). The World Cafe: Shaping our futures through conversations that matter. San Francisco, CA: Berrett-Koelher.

Brouwer, H., Woodhill, J, Hemmati M., Verhoosel, K, Vugt, S. V. (2019) The MSP Guide: How to design and facilitate multi-stakeholder partnerships. United Kingdom: Practical Action Publishing. 
Craik, F., \& Lockhart, R. (1972). Levels of processing: A framework for memory research. Journal of Verbal Learning and Verbal Behavior, 11, 671-684.

Cyrs, T. E. (1997). Visual thinking: let them see what you are saying. New directions for teaching and learning, n. 71, Fall, pp. 27-32.

Engelhardt, Y. (2002). The Language of Graphics: a framework for the analysis of syntax and meaning in maps, charts and diagrams. Amsterdam: ILLC- Publications.

Espiner, D.; \& Hartnett, F. (2016). Innovation and graphic facilitation. Aotearoa New Zealand Social Work Review, [s. I.], v. 28, n. 4, p. 44-53. DOI 10.11157/anzswj-vol28iss4id298.

Gardner, H. (1985). Frames of mind: Theory of multiple intelligences. New York, NY:Basic Books.

Gavron, T. (2013). Meeting on Common Ground: Assessing Parent-Child Relationships Through the Joint Painting Procedure, Art Therapy, 30:1, 12-19, DOI: 10.1080/07421656.2013.757508

Islim, O. F. (2018). Technology-supported collaborative concept maps in classrooms. Active Learning in Higher Education. v19, no. 2, p.131-143.

Knight, L., McArdle, F., Cumming, T., Bone, J., Li, L., Peterken, C., \& Ridgway, A. (2015). Intergenerational collaborative drawing: a research method for researching with/about young children. Australasian Journal of Early Childhood, vol. 40, no. 4, p. 21-29.

Lai, SS., Tsai, CH., Wu, CC., Chen, CT., Li,HJ., \& Chen, KL. (2020). Identifying the Cognitive Correlates of Reciprocity in Children with Autism Spectrum Disorder. J Autism Dev Disord 50, 2007-2018 (2020). https://doi.org/10.1007/s10803-019-03957-7

McKim, R.H. (1980). Experiences in visual thinking. Monterey, CA: Brooks/Cole Publishing Co.

Moreira, A. E. C., Oliveira, K. L., \& Scacchetti F. A. P. (2016). O processo de ensino e aprendizagem em questão: implicações metodológicas e motivacionais. Educação Unisinos. v. 20 , n. 1 , p. $106-116$.

Novak, J. D., \& Cañas, A. J. (2006). A teoria subjacente aos mapas conceituais e como elaborá-los e usá-los. Práxis Educativa, 5(1), 9-29.

Park, J., \& Kang, JR. (2018). Assessing the Six-Member Collaborative Drawing Technique with the Collaborative Art Elements Scale. Arts in Psychotherapy, v. 59, p. 75-82.

Prewitt, V. (2011). Working in the café: lessons in group dialogue. The Learning Organization, Vol. 18 No. 3, pp. 189-202.

Ravetz, J., \& Ravetz, A. (2017). Seeing the wood for the trees: Social Science 3.0 and the role of visual thinking. Innovation: The European Journal of Social Sciences, v. 30, n. 1, p. 104120. DOI 10.1080/13511610.2016.1224155.

Roam. D. (2009). The Back of the Napkin: Solving Problems and Selling Ideas with Pictures. Penguin Group, New York.

Rosen, M., Pitre, R., \& Johnson, D. R. (2016). Developmental Transformations Art Therapy: An Embodied, Interactional Approach, Art Therapy, 33:4, 195-202, DOI: 10.1080/07421656.2016.1229514 
Sakr, M. (2019). Young Children Drawing Together on the iPad Versus Paper: How Collaborative Creativity is Shaped by Different Semiotic Resources. International Journal of Education \& the Arts, v. 20 n 20. p.1-26.

Seels, B.A. (1994). Visual literacy: The definition problem. D.M. Moore ve F.M. Dwyer, (Eds.). Visual literacy: A spectrum of visual learning. (pp. 97-112). Englewood Cliffs, New Jersey: Educational Technology Publications.

Sibbet, D. (2001). A graphic facilitation retrospective. Adapted from a paper presented at the International Association of Facilitators: The Art and Mastery of Facilitation - Navigating the Future IAF Conference, 2001, May 16-20. Minnesota. DOI: https://doi.

org/10.1080/00405840801992306

Sibbet, D. (2013). Reuniões Visuais. Rio de Janeiro: Atlas Books.

Smith, G. M. (1985). The Collaborative Drawing Technique. Journal of Personality Assessment, [s. I.], v. 49 , n. 6 , p. 582 .

Snir S., \& Wiseman, H.(2010). Attachment in Romantic Couples and Perceptions of a Joint Drawing Session. The Family Journal. 18(2):116-126. doi:10.1177/1066480710364318

\section{Sobre os autores}

Luciana da Silva Bertoso, Me., UFPR, Brasil <lu.bertoso@gmail.com> Reinaldo Pereira de Moraes, Me., UFPR, Brasil <reinaldomoraes@ufpr.br> Stephania Padovani, Dra., UFPR, Brasil <stephania.padovani@gmail.com> 